\title{
Review Article \\ Fragility Fractures in Chronic Kidney Disease: Assessment and Pharmacologic Management
}

\author{
Mahesan Anpalahan, ${ }^{1,2}$ Sudharsan Venkatesan, ${ }^{3}$ and Aksharaa Anpalahan ${ }^{4}$ \\ ${ }^{1}$ Department of Internal Medicine, Eastern Health, Albert Street, Melbourne, VIC 3156, Australia \\ ${ }^{2}$ Department of Medicine, North West Academic Centre, University of Melbourne, St. Albans, Melbourne, VIC 3021, Australia \\ ${ }^{3}$ Division of Internal Medicine, Western Health, Gordon Street, Melbourne, VIC 3011, Australia \\ ${ }^{4}$ University of Melbourne, Melbourne, VIC 3010, Australia
}

Correspondence should be addressed to Mahesan Anpalahan; mahesan.anpalahan@easternhealth.org.au

Received 28 May 2014; Accepted 25 August 2014; Published 16 September 2014

Academic Editor: Francois Madore

Copyright (C) 2014 Mahesan Anpalahan et al. This is an open access article distributed under the Creative Commons Attribution License, which permits unrestricted use, distribution, and reproduction in any medium, provided the original work is properly cited.

Fractures are common in all stages of chronic kidney disease (CKD), and are associated with increased morbidity and mortality. Both CKD and osteoporosis often coexist as they both are strongly age associated. However, the management of fragility fractures in CKD poses many dilemmas. These include diagnosing the aetiology of fractures and choosing appropriate treatment. This paper reviews the current evidence for the assessment and pharmacologic management of fragility fractures in CKD.

\section{Introduction}

The burden of fracture has been shown to be high in all stages of chronic kidney disease (CKD). Studies have consistently shown that end stage kidney disease (ESKD) and dialysis are risk factors for low trauma fracture independent of age [1]. More recent studies have demonstrated that age adjusted prevalence of fracture is high even in early stages of CKD, including in those with age associated renal impairment [2]. Although the exact mechanisms responsible for the increased fracture risk in CKD have not been fully elucidated, it is not entirely unexpected as disorders of bone remodeling are observed as early as when eGFR is $<60 \mathrm{ml} / \mathrm{min} / 1.73 \mathrm{~m}^{2}$ [3]. However, the management of this common problem remains a challenge for many reasons. The diagnosis of the aetiopathology of fractures in CKD is not straightforward as, unlike in the general population, the fractures in CKD can be due to a heterogeneous group of bone disorders besides osteoporosis. Furthermore, the efficacy and safety of current osteoporosis therapies remain speculative in CKD.

\section{Diagnosis of Osteoporosis and Fracture Risk Assessment}

The current methods of diagnosing osteoporosis or predicting fractures either by the presence of a low trauma fracture or on the basis of bone mineral density (BMD) criteria (a T score of 2.5 standard deviations or more below the young adult mean BMD) by dual energy X-ray absorptiometry (DEXA) [4] have not been validated in chronic kidney disease (CKD). Although cross-sectional studies show that both dialysis and predialysis patients $[5,6]$ with fractures have a lower BMD compared with those without, fracture prediction by BMD has not been validated prospectively in CKD.

The biochemical abnormalities of CKD have now been recognized for their pathogenetic role not only in renal bone disease, often described as renal osteodystrophy (ROD), but also in a wide spectrum of disorders including vascular calcification and increased cardiovascular risk. As the definition of ROD does not adequately describe this diverse spectrum of disorders, the term chronic kidney disease-mineral bone 
disorder (CKD-MBD) has been coined to depict this multisystem disorder [7]. Renal osteodystrophy consists of the following group of disorders: a high bone turnover disease usually associated with secondary hyperparathyroidism; a low bone turnover disease that includes adynamic bone disease, osteomalacia, and aluminium bone disease; and mixed uraemic osteodystrophy. The term mixed uraemic osteodystrophy is used when morethan one subtype of these disorders are present on bone histomorphometry. Although osteomalacia is primarily a mineralisation disorder, it often has features of low bone turnover disease [8]. This is true for aluminium bone disease as well [8]. As low BMD may be associated with all subtypes of ROD, although relatively higher BMD has been reported with adynamic bone disease $[9,10]$, BMD by DEXA is unlikely to be sensitive or specific to either diagnose or differentiate osteoporosis from other disorders of ROD. Furthermore, the higher prevalence of abdominal aortic calcification and the differential effect of PTH on trabecular and cortical bones may also confound the interpretation BMD in CKD [9]. Other radiological modalities such as ultrahigh resolution peripheral quantitative computerized tomography (P-QCT) [11] and micro-MRI [12] are superior to DEXA by virtue of their ability to assess cortical and trabecular bone architecture. Although they show promise for fracture prediction in $\mathrm{CKD}$, they remain investigational at this stage.

Studies have investigated the associations of serum parathyroid hormone (PTH), bone specific alkaline phosphatase (BALP), and other bone turnover markers (BTMs) with $\mathrm{BMD}$, bone histomorphometry, and fractures in CKD $[10,13-$ 16]. The BTMs investigated include those that depend on renal clearance (serum $\mathrm{N}$ - and C-terminal cross-linking telopeptides of type 1 collagen (NTX, CTX), deoxypyridinoline, and osteocalcin) and others that are not (serum tartrateresistant acid phosphatase isoenzyme 5B (TRAP5b), Nterminal propeptide of type 1 collagen (PINP), and BALP [17]). In case of PINP, the trimeric or intact PINP, and not the total P1NP, appears to be free of renal clearance [18]. The BTMs, including those dependent on renal clearance, have been shown to discriminate high and low bone turnover states and fracture risk in some cross-sectional studies [13]. However, the overall results of these studies have been inconsistent and the clinical utility of BTMs other than for PTH and BALP has not been sufficiently validated in CKD. Serum PTH and BALP are useful for differentiating high and low bone turnover disease $[14,19,20]$. However, their diagnostic accuracy, especially for PTH is only modest, as the serum levels significantly overlap between high and low bone turnover states; thus only extreme values are diagnostically helpful in individual patients [16, 21]. Estimation of absolute fracture risk helps treatment decisions in clinical practice and fracture risk calculators based on BMD and validated clinical risk factors have been developed for this purpose. However, none of the currently available fracture risk assessment tools, including the FRAX [22] and Garvan Institute fracture risk calculator [23], make adjustments for fracture risk according to renal function, nor have they been validated in CKD.

At present, therefore, there are no high quality data available to formulate guidelines for fracture risk assessment in CKD. However, on the basis of available observational data and clinical expertise, consensus guidelines have been developed. In early stages of CKD (stages $1-3$ or eGFR $\geq 30 \mathrm{ml} / \mathrm{min} / 1.73 \mathrm{~m}^{2}$ ) the current WHO DEXA criteria may be used for diagnosing osteoporosis in the absence of abnormal serum bone biochemistry [7]. In more advanced stages of CKD (stage 4 and above), the diagnosis is more challenging, as evidence of abnormal bone metabolism is frequently evident when eGFR is $<30 \mathrm{ml} / \mathrm{min} / 1.73 \mathrm{~m}^{2}$ [24], and the diagnosis is best suggested by excluding ROD by quantitative bone histomorphometry. However, in selected patients with an eGFR $<30 \mathrm{ml} / \mathrm{min} / 1.73 \mathrm{~m}^{2}$, the current BMD criteria to diagnose osteoporosis may still be applied if there is no evidence of abnormal bone metabolism as evidenced by normal serum calcium, phosphate, ALP, and PTH in the absence of treatment with bone active agents such as phosphate binders, vitamin D metabolites, and calcimimetics.

\section{Pharmacologic Management of Low Trauma Fracture}

Although there is a plethora of literature on the pharmacologic management of osteoporosis in the general population, this is scarce in CKD patients. The pharmacologic management of bone fragility in CKD may be broadly classified into: (1) optimization of chronic kidney disease mineral bone disorder (CKD-MBD) and (2) the use of conventional osteoporosis therapies.

\section{Optimization of CKD-MBD}

The optimal management of CKD-MBD is likely to improve fracture risk in CKD, although there is no direct evidence for this. The current evidence suggests that fibroblast growth factor-23 (FGF-23) plays a central role in the pathogenesis of CKD-MBD [25]. Although we currently have only a fragmented understanding of its mode of action and control mechanisms, the current evidence suggests that it plays a significant role in secondary hyperparathyroidism and is the earliest biochemical abnormality in CKD-MBD [26]. Therefore, FGF 23 has emerged as a logical and an attractive therapeutic target in the management of CKD-MBD. However, to date, there is no firm evidence to suggest that modulating serum levels of FGF-23 will translate into improved clinical outcomes. Furthermore, animal studies have shown that administration of FGF-23 neutralizing antibodies has been associated with hyperphosphatemia, reduced bone turnover, vascular calcification, and increased mortality, although improvement in hyperparathyroidism has been noted [27].

The serum sclerostin, an osteocyte-derived bone morphogenetic protein antagonist, has been shown to correlate inversely with renal function [28], although its role in the pathogenesis of CKD-MBD has not been clearly defined. It could potentially play a role in the pathogenesis of adynamic bone disease as it inhibits osteoblastic activity. However, its clinical utility for differentiating high and low bone turnover states, or as a potential therapeutic target for managing low bone turnover states has not yet been explored. 
Therefore, at present, serum phosphate remains the earliest therapeutic target for managing CKD-MBD perhaps along with serum 25 -hydroxy vitamin $\mathrm{D}$. Therapeutic options for modulating hyperphosphatemia include dietary phosphate restriction, phosphate binders, and modification of dialysis techniques. The novel noncalcium based phosphate binders such as sevelamer and lanthanum carbonate may be superior to the calcium based binders as they are associated with less progression of vascular calcification and hypercalcaemia $[29,30]$, although the evidence for patient relevant cardiovascular or bone outcomes is limited [29, 30]. Aluminium based phosphate binders are potent phosphate binders but are not suitable for long-term use because of the risk of adynamic bone disease and aluminium toxicity $[8,31,32]$.

There are no fracture efficacy data for Vitamin D and calcium supplementation in CKD, especially in those with an eGFR $<30 \mathrm{ml} / \mathrm{min}$. However there is merit in correcting vitamin D deficiency to improve serum 25(OH)D levels to $50-75 \mathrm{nmols} /$ litre $(20-30 \mathrm{ng} / \mathrm{ml})$ as in the general population because of its established skeletal and extraskeletal effects [33]. The evidence linking calcium supplementation with increased cardiovascular risk in both CKD and general population should caution practitioners [34]. There is inadequate information on the optimum daily intake of calcium for CKD patients, but adverse events in the general population were reported with supplementation exceeding $1800 \mathrm{mg} /$ day [35]. Therefore, a total calcium intake of 1000-1200 mg/day, mostly from dietary sources, seems reasonable for most patients. Calcium based phosphate binders are a substantial source of elemental calcium and this should be factored into when estimating patients' daily calcium requirement.

The optimum serum PTH target in CKD is unclear and this is evidenced by the inconsistencies in the current guidelines [7, 36]. Methodological differences in PTH assay, paradigm shifts in treatment approaches to mineral bone disorders, and changing epidemiology of aluminium toxicity are among the potential confounders in assessing the literature. Most literature suggests that serum intact PTH levels below twice the upper normal reference and levels six or more times the upper normal reference are usually associated with low and high bone turnover, respectively, in dialysis patients [37]. However, these cutoffs should be used with the caveat that intact PTH levels between 2 and 9 times the normal reference range may not always correlate with bone histology or bone turnover states [7]. Defining the optimal PTH target for predialysis patients is even more difficult. The KDIGO guideline suggests maintaining the serum intact PTH in the normal reference range for CKD stages 3-5 not on dialysis and recommends evaluation and management if there is progressive rise in serum intact PTH above the upper normal limit [7]. However, these recommendations are not based on high quality evidence and maintaining normal serum PTH levels in CKD stages 4 and 5 may predispose to low bone turnover disease [37]. The KDOQI guideline probably reflects this concern and recommends serum PTH targets based on the stage of the CKD in predialysis patients [36]. The pharmacological agents that are useful for controlling secondary hyperparathyroidism include calcium and noncalcium based phosphate binders, vitamin $\mathrm{D}$, vitamin $\mathrm{D}$ analogues and calcimimetics. The advent of calcimimetics has revolutionized the management of secondary hyperparathyroidism, and the use of cinacalcet has been associated with a reduction in parathyroidectomy and fractures in dialysis patients [38].

\section{The Clinical Utility of Osteoporosis Therapies in CKD}

The safety and efficacy of conventional osteoporosis therapies have not been adequately validated in CKD. The bisphosphonates, the commonly used antiresorptives, are predominantly dependent on renal clearance [39] and their intravenous formulations are potentially nephrotoxic [40]. Furthermore, antiresorptives pose a potential risk for low turnover bone disease, a common form of ROD [41]. The manufacturer's recommendations for the use of currently approved osteoporosis therapies in renal impairment are summarized in Table 1.

Bisphosphonates. A significant renal impairment has generally been an exclusion criterion in clinical trials of osteoporosis therapies. For example, the serum creatinine cutoffs in the alendronate trials had been $>130 \mathrm{mmols} / 1(>1.5 \mathrm{mg} / \mathrm{dl})$ [42] and $>144 \mathrm{mmols} / 1$ ( $>1.6 \mathrm{mg} / \mathrm{dl})$ [43]. Therefore, prospective data are scarce for the clinical utility of bisphosphonates in CKD. However, there is a substantial body of information available from post hoc analyses of major osteoporosis drug trials for the use of alendronate and risedronate in CKD patients at least in the context of normal bone biochemistry $[44,45]$. These data show that alendronate $10 \mathrm{mg}$ daily is generally safe and effective in CKD stages 1-3 for a duration up to 4 years, at least in the setting of age related renal impairment. There is also limited information for the efficacy of alendronate in CKD Stage 4 from these analyses. The efficacy and safety data for risedronate in CKD come from the pooled data of nine randomized controlled trials [44]. The renal function in this analysis was determined by estimating creatinine clearance $(\mathrm{CrCl})$ using the Cockcroft, Gault formula. $45 \%$ of participants in this analysis had a $\mathrm{CrCl}$ between 30 and $50 \mathrm{ml} / \mathrm{min}$ and $7 \%$ had a $\mathrm{CrCl}<30 \mathrm{ml} / \mathrm{min}$, and treatment with $5 \mathrm{mg}$ risedronate daily up to three years was found to be safe and effective. The recent clinical trials of intravenous bisphosphonates such as zoledronic acid have used a $\mathrm{CrCl}(<30 \mathrm{ml} / \mathrm{min})$ rather than a serum creatinine cutoff for exclusion [46]. Therefore, unlike the oral bisphosphonates, there are no fracture efficacy data available for zoledronic acid in stage 4 CKD. A transient rise in serum creatinine has been observed in a small but a significant number of patients receiving zoledronic acid infusion [47] and, as with the intravenous administration of other bisphosphonates such as etidronate, clodronate, tiludronate, and ibandronate, a rapid infusion rate appears to exacerbate this [48]. Therefore, an infusion rate of at least $>15$ minutes, preferably 60 minutes, is recommended in CKD and zoledronic acid is contraindicated in patients with a $\mathrm{CrCl}$ $<35 \mathrm{ml} / \mathrm{min}$ [49]. Ibandronate is another bisphosphonate available for intravenous administration in patients with a $\mathrm{CrCl}>30 \mathrm{ml} / \mathrm{min}$ [50]. It is, however, less widely used for 
TABLE 1: The manufacturer's recommendations for the use of currently approved osteoporosis therapies in renal impairment.

\begin{tabular}{lll}
\hline Osteoporosis therapy & $\begin{array}{l}\text { Creatinine } \\
(\mathrm{mmol} / \mathrm{L})\end{array}$ & $\begin{array}{l}\mathrm{CrCL} \\
(\mathrm{mL} / \mathrm{min})\end{array}$ \\
\hline Alendronate & $>35$ \\
Risedronate & $>30$ \\
Zoledronic acid & $>35$ \\
Ibandronate & $>50-$ for CrCL below 50; manufacturer advises reduction in dose \\
Raloxifene & Dose adjustment not required \\
Strontium ranelate & $>30$ \\
Denosumab & $>30-$ for CrCL below 30 or receiving dialysis; manufacturer warns of risk of hypocalcaemia \\
Teriparatide & & \\
Calcitonin & $<177$ & Mild dose adjustment in renal impairment \\
\hline
\end{tabular}

postmenopausal osteoporosis as its fracture efficacy data are relatively less robust when compared with zoledronic acid, although there are no head to head data available.

\section{Other Osteoporosis Pharmacologic Agents}

Raloxifene. As with oral bisphosphonates, there is evidence from post hoc analyses for the use of raloxifene in CKD stages 1-4, although the data are quite limited in CKD stage 4 [51]. Raloxifene, however, is not the usual first line therapy for osteoporosis as it is a less potent antiresorptive agent in that the registration trial showed no benefit for reduction of nonvertebral or hip fracture.

Teriparatide. A serum creatinine $>177 \mathrm{mmols} / \mathrm{l}(2 \mathrm{mg} / \mathrm{dl})$ and an abnormal serum PTH were exclusion criteria in teriparatide clinical trials. However, post hoc analysis suggests that teriparatide is effective as measured by an increase in BMD and P1NP and safe in patients with CrCL down to $30 \mathrm{ml} / \mathrm{min}$ in the setting of normal serum PTH. As there were only a very few patients in the $\mathrm{CrCL}<30 \mathrm{ml} / \mathrm{min}$ cohort, any meaningful interpretation of the data is difficult in stage 4 CKD [52]. Teriparatide may also have a therapeutic role in low bone turnover adynamic bone disease [53].

Denosumab. Unlike other antiresorptives, denosumab is not dependent on renal clearance, and, therefore, renal impairment is not a contraindication for its administration [54, 55]. However, at present, the fracture efficacy data for this agent in CKD is available only from post hoc analysis [55]. The secondary analysis of the FREEDOM trial [56] suggests that denosumab is safe and effective in patients with an eGFR down to $15 \mathrm{ml} / 1.73 \mathrm{~m}^{2}$. However, the analysis could not confirm fracture efficacy in stage 4 CKD due to lack of statistical power, although there was bone density response. As with other antiresorptives, low bone turnover should be a consideration for denosumab. In addition, caution should be exercised to prevent hypocalcaemia, as the risk of hypocalcaemia is greater for CKD patients receiving denosumab [57].

Strontium Ranelate, Calcitonin, and Gonadal Hormones. There are limited data available for their use in CKD, although they appear safe in CKD stages $1-3[58,59]$. The recent findings from the pooled analysis of RCTs of strontium suggesting an increased cardiovascular risk renders this drug less appealing for use in CKD. Calcitonin is metabolized and excreted predominantly by kidneys and the manufacturer's labeling recommends dose adjustment, although the details are not clear [58]. Hormone replacement therapy (HRT) is no longer considered a 1st-line therapy for postmenopausal osteoporosis. However, HRT may be an option in some premenopausal women with CKD and secondary amenorrhoea. Similarly, testosterone replacement may be considered in selected men with CKD and hypogonadism, although this has not been specifically studied in CKD.

There are no efficacy data to support the use of currently approved osteoporosis therapies in CKD stage 5 and 5D. In general, treatment decisions will have to be individualized, and anecdotally bisphosphonates may be considered in stages 5 and 5D in those with fractures after exclusion of adynamic bone disease and osteomalacia by undertaking a bone biopsy. It is suggested that half the usual dose prescribed for osteoporosis is used for no more than three years in these patients [60].

\section{Conclusion}

The management of fragility fractures in CKD presents unique challenges - an area that bounds with opinion but with limited evidence. On the basis of available evidence BMD by DEXA appears valid for fracture risk assessment in early stages of CKD down to stage 3 in the absence of biochemical abnormalities that may suggest ROD. The use of fracture risk calculators such as FRAX may also be valid in early stages of CKD. In more advanced stages of CKD the diagnosis is more challenging and would require exclusion of ROD. It would appear that all drugs that are currently approved for the treatment of postmenopausal osteoporosis can be safely used without dose adjustment in CKD stages 1-3, at least for up to three years. The clinical utility of these drugs in late stages of CKD is unknown. Furthermore, treatment of low bone turnover disease is difficult; antiresorptives are contraindicated and there is only anecdotal evidence for the use of anabolic agents. Therefore, there is a need for testing 
novel therapies for bone protection in CKD. An ideal therapeutic agent perhaps should be nonnephrotoxic, not renally cleared, and have dual action on both bone formation and resorption, so that it will have therapeutic appeal for both high and low bone turnover states. Organic nitrates appear to fulfill many of these attributes. Nitrates have been shown to be effective for bone protection in postmenopausal osteoporosis [61], although no data are available in CKD. Romosozumab, a humanised antisclerostin monoclonal antibody, is another osteoporosis therapeutic agent that may hold promise for ROD, particularly for low bone turnover disease. Although romosozumab has not yet been specifically tested in CKD population, the recently published phase 2 clinical trial recruited patients with eGFR down to $30 \mathrm{ml} /$ minute [62]. Overall, the management of bone disease in CKD, particularly in late stages, CKD-4-5/5D, requires further research.

\section{Conflict of Interests}

The authors declare that there is no conflict of interests regarding the publication of this paper.

\section{References}

[1] A. M. Alem, D. J. Sherrard, D. L. Gillen et al., "Increased risk of hip fracture among patients with end-stage renal disease," Kidney International, vol. 58, no. 1, pp. 396-399, 2000.

[2] T. L. Nickolas, D. J. McMahon, and E. Shane, "Relationship between moderate to severe kidney disease and hip fracture in the United States," Journal of the American Society of Nephrology, vol. 17, no. 11, pp. 3223-3232, 2006.

[3] A. Levin, G. L. Bakris, M. Molitch et al., "Prevalence of abnormal serum vitamin $\mathrm{D}, \mathrm{PTH}$, calcium, and phosphorus in patients with chronic kidney disease: results of the study to evaluate early kidney disease," Kidney International, vol. 71, no. 1, pp. 31-38, 2007.

[4] J. A. Kanis and J. A. Kanis, "Assessment of fracture risk and its application to screening for postmenopausal osteoporosis: Synopsis of a WHO report," Osteoporosis International, vol. 4, no. 6, pp. 368-381, 1994.

[5] T. L. Nickolas, E. Stein, A. Cohen et al., "Bone mass and microarchitecture in CKD patients with fracture," Journal of the American Society of Nephrology, vol. 21, no. 8, pp. 1371-1380, 2010.

[6] S. A. Jamal, J. A. Hayden, and J. Beyene, "Low bone mineral density and fractures in long-term hemodialysis patients: a meta-analysis," The American Journal of Kidney Diseases, vol. 49, no. 5, pp. 674-681, 2007.

[7] "KDIGO clinical practice guideline for the diagnosis, evaluation, prevention, and treatment of Chronic Kidney DiseaseMineral and Bone Disorder (CKD-MBD)," Kidney International. Supplement, vol. 113, pp. S1-S130, 2009.

[8] V. M. Brandenburg and J. Floege, "Adynamic bone diseasebone and beyond," NDT Plus, vol. 1, no. 3, pp. 135-147, 2008.

[9] C. Ambrus, A. Marton, Z. K. Nemeth, and I. Mucsi, "Bone mineral density in patients on maintenance dialysis," International Urology and Nephrology, vol. 42, no. 3, pp. 723-739, 2010.

[10] A. Gerakis, D. Hadjidakis, E. Kokkinakis, T. Apostolou, S. Raptis, and A. Billis, "Correlation of bone mineral density with the histological findings of renal osteodystrophy in patients on hemodialysis," Journal of Nephrology, vol. 13, no. 6, pp. 437-443, 2000.

[11] J. Bacchetta, S. Boutroy, N. Vilayphiou et al., "Early impairment of trabecular microarchitecture assessed with HR-pQCT in patients with stage II-IV chronic kidney disease," Journal of Bone and Mineral Research, vol. 25, no. 4, pp. 849-857, 2010.

[12] F. W. Wehrli, M. B. Leonard, P. K. Saha, and B. R. Gomberg, "Quantitative high-resolution magnetic resonance imaging reveals structural implications of renal osteodystrophy on trabecular and cortical bone," Journal of Magnetic Resonance Imaging, vol. 20, no. 1, pp. 83-89, 2004.

[13] T. L. Nickolas, S. Cremers, A. Zhang et al., "Discriminants of prevalent fractures in chronic kidney disease," Journal of the American Society of Nephrology, vol. 22, no. 8, pp. 1560-1572, 2011.

[14] P. Urena and M.-C. de Vernejoul, "Circulating biochemical markers of bone remodeling in uremic patients," Kidney International, vol. 55, no. 6, pp. 2141-2156, 1999.

[15] G. Lehmann, U. Ott, D. Kämmerer, J. Schütze, and G. Wolf, "Bone histomorphometry and biochemical markers of bone turnover in patients with chronic kidney disease Stages 3-5," Clinical Nephrology, vol. 70, no. 4, pp. 296-305, 2008.

[16] A. R. J. Bervoets, G. B. Spasovski, G. J. Behets et al., "Useful biochemical markers for diagnosing renal osteodystrophy in predialysis end-stage renal failure patients," The American Journal of Kidney Diseases, vol. 41, no. 5, pp. 997-1007, 2003.

[17] R. Swaminathan, "Biochemical markers of bone turnover," Clinica Chimica Acta, vol. 313, no. 1-2, pp. 95-105, 2001.

[18] J. H. Krege, N. E. Lane, J. M. Harris et al., "PINP as a biological response marker during teriparatide treatment for osteoporosis," Osteoporosis International: A Journal Established as Result of Cooperation between the European Foundation for Osteoporosis and the National Osteoporosis Foundation of the USA, vol. 25, no. 9, pp. 2159-2171, 2014.

[19] Q. Qi, M.-C. Monier-Faugere, Z. Geng, and H. H. Malluche, "Predictive value of serum parathyroid hormone levels for bone turnover in patients on chronic maintenance dialysis," The American Journal of Kidney Diseases, vol. 26, no. 4, pp. 622-631, 1995.

[20] M. M. Couttenye, P. C. D’Haese, V. O. van Hoof et al., "Low serum levels of alkaline phosphatase of bone origin: a good marker of adynamic bone disease in haemodialysis patients," Nephrology Dialysis Transplantation, vol. 11, no. 6, pp. 10651072, 1996.

[21] A. Gal-Moscovici and M. M. Popovtzer, "New worldwide trends in presentation of renal osteodystrophy and its relationship to parathyroid hormone levels," Clinical Nephrology, vol. 63, no. 4, pp. 284-289, 2005.

[22] J. A. Kanis, O. Johnell, A. Oden, H. Johansson, and E. McCloskey, "FRAXUं and the assessment of fracture probability in men and women from the UK," Osteoporosis International, vol. 19, no. 4, pp. 385-397, 2008.

[23] N. D. Nguyen, S. A. Frost, J. R. Center, J. A. Eisman, and T. V. Nguyen, "Development of prognostic nomograms for individualizing 5-year and 10-year fracture risks," Osteoporosis International, vol. 19, no. 10, pp. 1431-1444, 2008.

[24] K. E. Ensrud, L.-Y. Lui, B. C. Taylor et al., "Renal function and risk of hip and vertebral fractures in older women," Archives of Internal Medicine, vol. 167, no. 2, pp. 133-139, 2007.

[25] T. E. Larsson, "The role of FGF-23 in CKD-MBD and cardiovascular disease: friend or foe?” Nephrology Dialysis Transplantation, vol. 25, no. 5, pp. 1376-1381, 2010. 
[26] T. Isakova, P. Wahl, G. S. Vargas et al., "Fibroblast growth factor 23 is elevated before parathyroid hormone and phosphate in chronic kidney disease," Kidney International, vol. 79, no. 12, pp. 1370-1378, 2011.

[27] V. Shalhoub, E. M. Shatzen, S. C. Ward et al., "FGF23 neutralization improves chronic kidney disease-associated hyperparathyroidism yet increases mortality," Journal of Clinical Investigation, vol. 122, no. 7, pp. 2543-2553, 2012.

[28] S. Pelletier, L. Dubourg, M.-C. Carlier, A. Hadj-Aissa, and D. Fouque, "The relation between renal function and serum sclerostin in adult patients with CKD," Clinical Journal of the American Society of Nephrology, vol. 8, no. 5, pp. 819-823, 2013.

[29] M. Tonelli, N. Wiebe, B. Culleton et al., "Systematic review of the clinical efficacy and safety of sevelamer in dialysis patients," Nephrology Dialysis Transplantation, vol. 22, no. 10, pp. 28562866, 2007.

[30] A. J. Hutchison, B. Maes, J. Vanwalleghem et al., "Efficacy, tolerability, and safety of lanthanum carbonate in hyperphosphatemia: a 6-month, randomized, comparative trial versus calcium carbonate," Nephron: Clinical Practice, vol. 100, no. 1, pp. c8-c19, 2005.

[31] J. B. Cannata-Andia, J. T. Harrington, M. Martinez-Maldonado et al., "Hypokinetic azotemic osteodystrophy," Kidney International, vol. 54, no. 3, pp. 1000-1016, 1998.

[32] A. C. Alfrey, A. Hegg, and P. Craswell, "Metabolism and toxicity of aluminum in renal failure," The American Journal of Clinical Nutrition, vol. 33, no. 7, pp. 1509-1516, 1980.

[33] R. Rizzoli, S. Boonen, M. L. Brandi et al., "Vitamin D supplementation in elderly or postmenopausal women: a 2013 update of the 2008 recommendations from the European Society for Clinical and Economic Aspects of Osteoporosis and Osteoarthritis (ESCEO)," Current Medical Research and Opinion, vol. 29, no. 4, pp. 305-313, 2013.

[34] S. L. West, V. J. Swan, and S. A. Jamal, "Effects of calcium on cardiovascular events in patients with kidney disease and in a healthy population," Clinical Journal of the American Society of Nephrology, vol. 5, supplement 1, pp. S41-S47, 2010.

[35] M. J. Bolland, P. A. Barber, R. N. Doughty et al., "Vascular events in healthy older women receiving calcium supplementation: randomised controlled trial," BMJ, vol. 336, no. 7638, pp. 262266, 2008.

[36] "K/DOQI clinical practice guidelines for bone metabolism and disease in chronic kidney disease," American Journal of Kidney Diseases, vol. 42, no. 4, supplement 3, pp. S1-S201, 2003.

[37] A. Torres, V. Lorenzo, D. Hernandez et al., "Bone disease in predialysis, hemodialysis, and CAPD patients: evidence of a better bone response to PTH," Kidney International, vol. 47, no. 5, pp. 1434-1442, 1995.

[38] J. Cunningham, M. Danese, K. Olson, P. Klassen, and G. M. Chertow, "Effects of the calcimimetic cinacalcet $\mathrm{HCl}$ on cardiovascular disease, fracture, and health-related quality of life in secondary hyperparathyroidism," Kidney International, vol. 68, no. 4, pp. 1793-1800, 2005.

[39] J. H. Lin, "Bisphosphonates: a review of their pharmacokinetic properties," Bone, vol. 18, no. 2, pp. 75-85, 1996.

[40] P. Conte and V. Guarneri, "Safety of intravenous and oral bisphosphonates and compliance with dosing regimens," The Oncologist, vol. 9, supplement 4, pp. 28-37, 2004.

[41] D. J. Sherrard, G. Hercz, Y. Pei et al., "The spectrum of bone disease in end-stage renal failure - an evolving disorder," Kidney International, vol. 43, no. 2, pp. 436-442, 1993.
[42] U. A. Liberman, S. R. Weiss, J. Bröll et al., "Effect of oral alendronate on bone mineral density and the incidence of fractures in postmenopausal osteoporosis," New England Journal of Medicine, vol. 333, no. 22, pp. 1437-1443, 1995.

[43] D. M. Black, S. R. Cummings, D. B. Karpf et al., "Randomised trial of effect of alendronate on risk of fracture in women with existing vertebral fractures," The Lancet, vol. 348, no. 9041, pp. 1535-1541, 1996.

[44] P. D. Miller, C. Roux, S. Boonen, I. P. Barton, L. E. Dunlap, and D. E. Burgio, "Safety and efficacy of risedronate in patients with age-related reduced renal function as estimated by the Cockcroft and Gault method: a pooled analysis of nine clinical trials," Journal of Bone and Mineral Research, vol. 20, no. 12, pp. 2105-2115, 2005.

[45] S. A. Jamal, D. C. Bauer, K. E. Ensrud et al., "Alendronate treatment in women with normal to severely impaired renal function: an analysis of the fracture intervention trial," Journal of Bone and Mineral Research, vol. 22, no. 4, pp. 503-508, 2007.

[46] D. M. Black, P. D. Delmas, R. Eastell et al., "Once-yearly zoledronic acid for treatment of postmenopausal osteoporosis," The New England Journal of Medicine, vol. 356, no. 18, pp. 18091822, 2007.

[47] S. Boonen, D. E. Sellmeyer, K. Lippuner et al., "Renal safety of annual zoledronic acid infusions in osteoporotic postmenopausal women," Kidney International, vol. 74, no. 5, pp. 641-648, 2008.

[48] P. D. Miller, P. Ward, T. Pfister, C. Leigh, and J.-J. Body, "Renal tolerability of intermittent intravenous ibandronate treatment for patients with postmenopausal osteoporosis: a review," Clinical and Experimental Rheumatology, vol. 26, no. 6, pp. 1125-1133, 2008.

[49] P. D. Miller, “The kidney and bisphosphonates," Bone, vol. 49, no. 1, pp. 77-81, 2011.

[50] P. D. Miller, S. Ragi-Eis, C. Mautalen, F. Ramirez, and I. Jonkanski, "Effects of intravenous ibandronate injection on renal function in women with postmenopausal osteoporosis at high risk for renal disease: the DIVINE study," Bone, vol. 49, no. 6, pp. 1317-1322, 2011.

[51] A. Ishani, T. Blackwell, S. A. Jamal, S. R. Cummings, and K. E. Ensrud, "The effect of raloxifene treatment in postmenopausal women with CKD," Journal of the American Society of Nephrology, vol. 19, no. 7, pp. 1430-1438, 2008.

[52] P. D. Miller, E. N. Schwartz, P. Chen, D. A. Misurski, and J. H. Krege, "Teriparatide in postmenopausal women with osteoporosis and mild or moderate renal impairment," Osteoporosis International, vol. 18, no. 1, pp. 59-68, 2007.

[53] D. Cejka, K. Kodras, T. Bader, and M. Haas, "Treatment of hemodialysis-associated adynamic bone disease with teriparatide (PTH1-34): a pilot study," Kidney and Blood Pressure Research, vol. 33, no. 3, pp. 221-226, 2010.

[54] G. A. Block, H. G. Bone, L. Fang, E. Lee, and D. Padhi, "A singledose study of denosumab in patients with various degrees of renal impairment," Journal of Bone and Mineral Research, vol. 27, no. 7, pp. 1471-1479, 2012.

[55] S. A. Jamal, Ö. Ljunggren, C. Stehman-Breen et al., "Effects of denosumab on fracture and bone mineral density by level of kidney function," Journal of Bone and Mineral Research, vol. 26, no. 8, pp. 1829-1835, 2011.

[56] S. R. Cummings, J. S. Martin, M. R. McClung et al., "Denosumab for prevention of fractures in postmenopausal women with osteoporosis," New England Journal of Medicine, vol. 361, no. 8, pp. 756-765, 2009. 
[57] B. B. Mccormick, J. Davis, and K. D. Burns, "Severe hypocalcemia following denosumab injection in a hemodialysis patient," The American Journal of Kidney Diseases, vol. 60, no. 4, pp. 626-628, 2012.

[58] Novartis, "Salcatonin prescribing information," http://www.novartis.com.au/pi_pdf/mia.pdf.

[59] Servier, Protos Prescribing Information, 2013, http://www.servier.co.uk/healthcare-professionals/protelos/references.asp.

[60] P. D. Miller, "Treatment of osteoporosis in chronic kidney disease and end-stage renal disease," Curr Osteoporos Rep, vol. 3, no. 1, pp. 5-12, 2005.

[61] S. A. Jamal, C. J. Hamilton, R. Eastell, and S. R. Cummings, "Effect of nitroglycerin ointment on bone density and strength in postmenopausal women a randomized trial," The Journal of the American Medical Association, vol. 305, no. 8, pp. 800-807, 2011.

[62] M. R. McClung, A. Grauer, and S. Boonen, "Romosozumab in women with low bone mineral density," The New England Journal of Medicine, vol. 370, no. 5, pp. 412-420, 2014. 


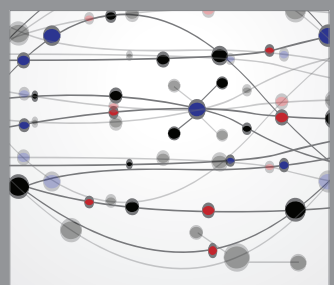

The Scientific World Journal
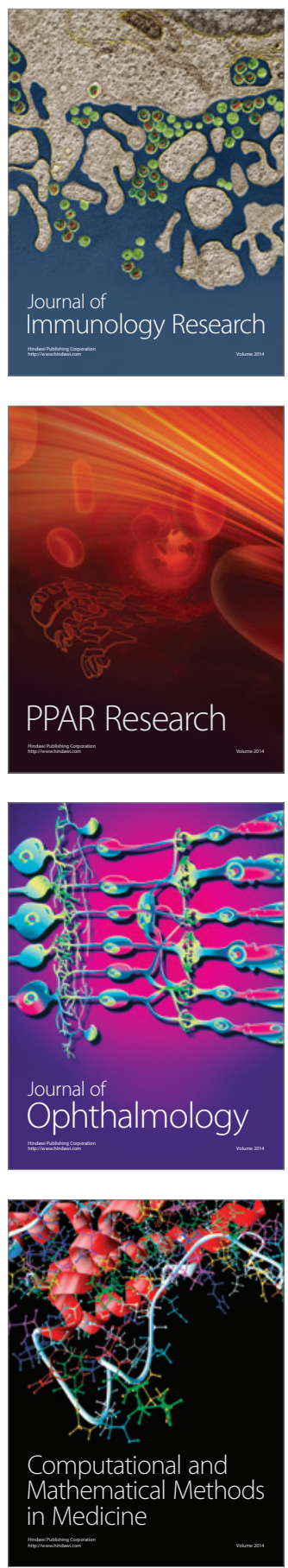

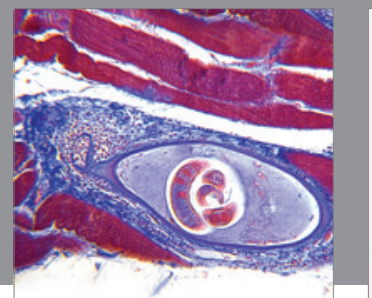

Gastroenterology

Research and Practice
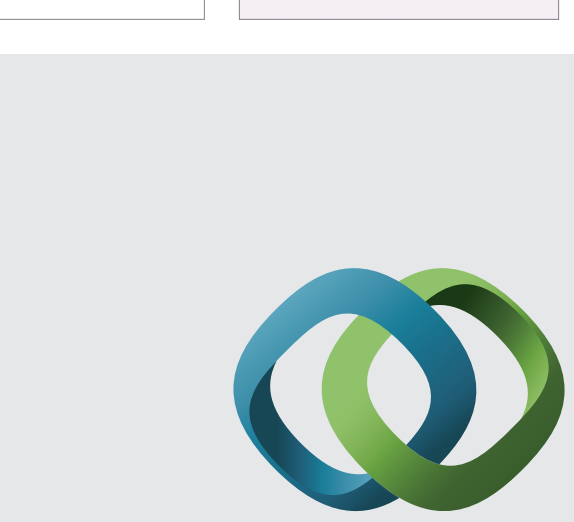

\section{Hindawi}

Submit your manuscripts at

http://www.hindawi.com
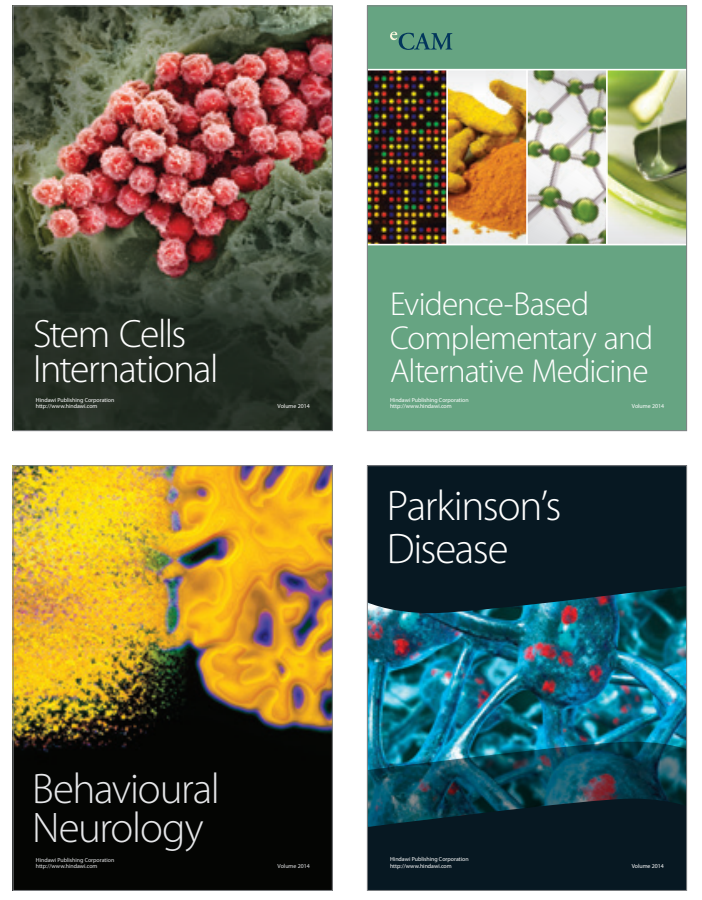
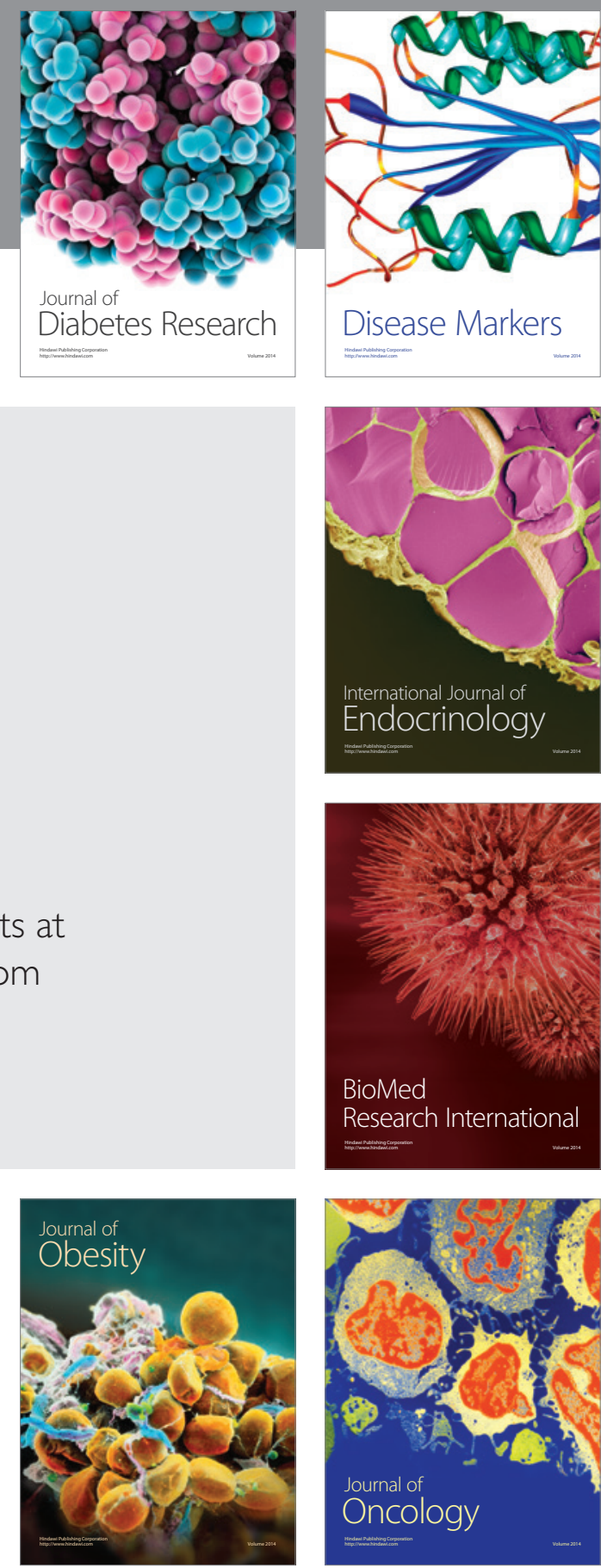

Disease Markers
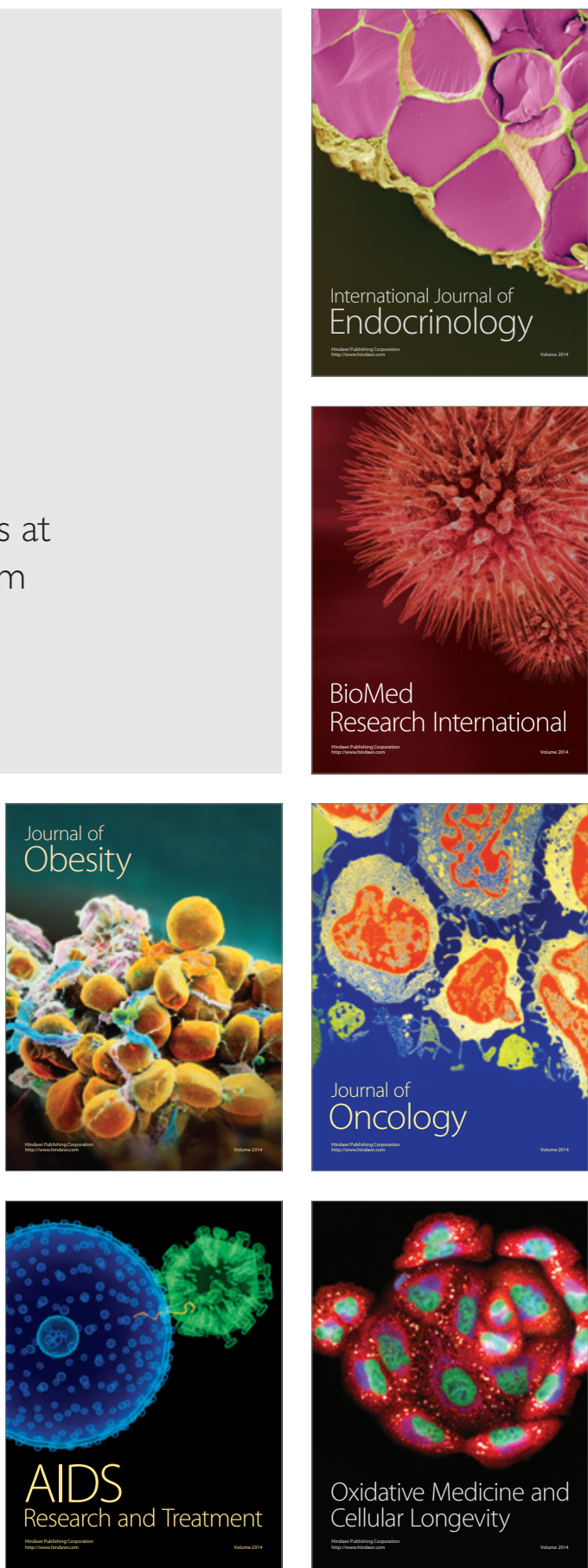